\title{
Observation of second-order spectral phase transition in optical parametric oscillator
}

\author{
Arkadev Roy $^{1}$, Saman Jahani ${ }^{1}$, Carsten Langrock ${ }^{2}$, Martin Fejer ${ }^{2}$, Alireza Marandi ${ }^{1}$ \\ ${ }^{1}$ Electrical Engineering Department, California Institute of Technology, Pasadena, California, 91125, USA \\ ${ }^{2}$ Edward L. Ginzton Laboratory, Stanford University, Stanford, California, 94305, USA \\ marandi@caltech.edu
}

\begin{abstract}
We demonstrate second-order phase transition in optical parametric oscillators corresponding to a transition from degenerate to non-degenerate oscillation at a critical cavity detuning which can be utilized for enhanced sensing, and information processing. (c) 2020 The Author(s)
\end{abstract}

Driven dissipative systems in non-equilibrium steady states exhibit a wide range of intriguing phenomena including self-organization, pattern formation, emergent phase and dynamical phase transition [1], and exceptional point arising from PT-symmetry breaking [2]. Driven nonlinear optical resonators provide a fertile ground to explore non-equilibrium physics [3], however, such systems are not extensively studied in the spectral domain. Here, we show that an optical parametric oscillator (OPO) near degeneracy can undergo a dynamical phase transition from its degenerate (ordered state) to non-degenerate (disordered) state by cavity detuning. We define and analyze the variation of an order parameter as a function of cavity detuning, and experimentally and numerically show a rapid (square-root) spectral splitting as a function of the detuning.

We consider a doubly-resonant OPO, where the signal and the idler are resonant in a lossy dispersive cavity, with a pump at twice the frequency of the mean of the signal and idler frequencies [4]. We observe a secondorder spectral phase transition at a critical cavity detuning corresponding to a transition from degenerate to nondegenerate optical parametric oscillation. This novel nonlinear phenomena of spectral self-organization adopted by the system in the pursuit of minimizing the threshold belongs to the class of dynamical phase transitions and can have myriad applications.

In the $\mathrm{CW}$-driven, high-finesse limit, the $\mathrm{OPO}$ is governed by the mean-field evolution equation:

$$
\frac{\partial a}{\partial \xi}=(-\alpha+i \Delta \phi) a+g a^{*}-i \frac{\beta_{2}}{2} \frac{\partial^{2} a}{\partial t^{2}}-\left[\frac{\varepsilon^{2}}{2 u^{2}} \int_{0}^{L u}(L u-\tau) a(t-\tau)^{2} d \tau\right] a^{*},
$$

where, $a$ describes the signal envelope under the slowly varying envelope approximation limit. Here $\xi, t$ refers to the slow time and the fast time, respectively. $\alpha, \Delta \phi, \beta_{2}$, and $g$ denote the loss, detuning, second-order group velocity dispersion (GVD), and the phase-sensitive parametric gain, respectively. $g$ in the CW-limit is expressed as $\varepsilon b L$, where $b$ is the pump amplitude. $L$ refers to the cavity round trip length where the nonlinear interaction is encountered, $\varepsilon$ includes the strength of the nonlinear interaction and $u$ is the walk-off parameter. The last term to the right of the equation is responsible for the gain saturation. Each of these terms are normalized by suitable normalization factors.

We assume a perturbation in the signal around the half-harmonic frequency to be of the form: $a=a_{+} e^{i \delta \omega t}+$ $a_{-} e^{-i \delta \omega t}$. Linear stability analysis to determine the most unstable longitudinal mode gives:

$$
\begin{gathered}
\frac{d}{d \xi}\left[\begin{array}{l}
a_{+} \\
a_{-}^{*}
\end{array}\right]=\left[\begin{array}{cc}
-\alpha+i \Delta \phi+i \frac{\beta_{2}}{2}(\delta \omega)^{2} & g \\
g^{*} & -\alpha-i \Delta \phi-i \frac{\beta_{2}}{2}(\delta \omega)^{2}
\end{array}\right]\left[\begin{array}{l}
a_{+} \\
a_{-}^{*}
\end{array}\right] \\
\lambda_{ \pm}=-1 \pm \sqrt{|g|^{2}-\left(\Delta \phi+\beta_{2}(\delta \omega)^{2}\right)^{2}}
\end{gathered}
$$

By analyzing the eigenvalue (growth rate)(Eq(2b)) of the linear stability matrix we arrive at two scenarios. When $\operatorname{sgn}(\Delta \phi)=\operatorname{sgn}\left(\beta_{2}\right)$, we find that the most unstable frequency of oscillation is $\delta \omega=0$, and the corresponding threshold (i.e. when $\lambda_{+}=0$ ) is $|g|_{t h}=\sqrt{\alpha^{2}+(\Delta \phi)^{2}}$, leaving the OPO in the degenerate phase. When $\operatorname{sgn}(\Delta \phi)=$ $-\operatorname{sgn}\left(\beta_{2}\right)$, the most unstable frequency of oscillation is given by $\delta \omega= \pm\left|\frac{2 \Delta \phi}{\beta_{2}}\right|^{\frac{1}{2}}$, and the associated threshold is $|g|_{t h}=\alpha$, leaving the OPO in the non-degenerate phase. This proves the existence of the spectral phase transition.

The spectral splitting $\left(\delta \omega= \pm\left|\frac{2 \Delta \phi}{\beta_{2}}\right|^{\frac{1}{2}}\right)$ in the non-degenerate phase shows square-root behavior with detuning. This is akin to what is exhibited by exceptional points arising in the context of non-Hermitian degeneracies and has been utilized for enhanced sensitivity [5]. The square-root behaviour around the critical point results in a large 
(a)

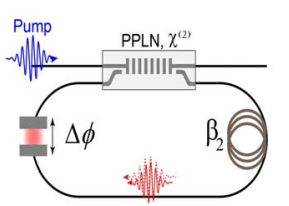

(b)

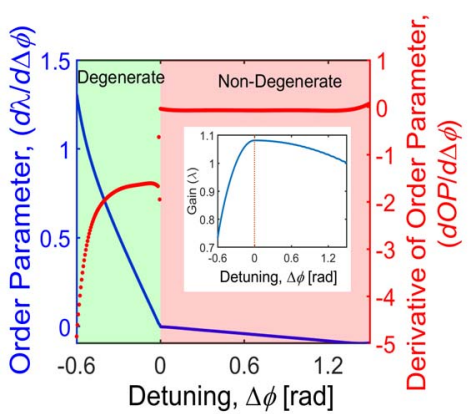

(c)

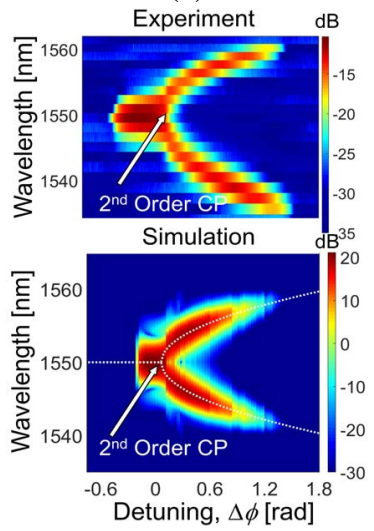

(d)

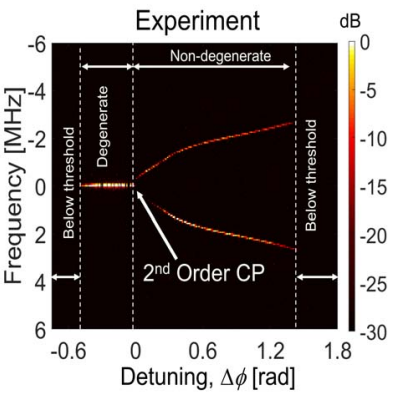

Fig 1. Second-order spectral phase transition in OPO. a) Schematic of the experimental setup. b) Order parameter as a function of detuning; continuous order parameter but discontinuous derivative atypical of a second order phase transition is plotted. The inset shows the gain curve as a function of cavity detuning. The gain curve has its maximum at the critical point (CP). c) Spectrum as a function of detuning: Experimental results (top) capturing the second-order critical point, numerical simulation result (bottom) (dotted line plots the theoretically expected spectral splitting). It closely follows the square-root behaviour in the non-degenerate regime. d) Emergence of the carrier-envelope-offset frequencies of the signal and idler around the critical point. Measured RF spectrum captured using a fast detector as a result of interfering the OPO output and a local oscillator.

sensitivity when biased around the critical point. Thus our work can pave the way for the development of critical/ transition edge sensors leveraging the phenomenon of spectral phase transition.

Figure 1a shows the experimental setup which comprises a periodically poled lithium niobate (PPLN) waveguide which provides parametric gain around $1550 \mathrm{~nm}$ using a pump around $775 \mathrm{~nm}$ [6]. The resonator is formed using polarization maintaining fiber and a free-space portion, which can provide precise detuning $(\Delta \phi)$ using a PZT. The OPO is pumped by $\sim 15$-ps pulses $(775 \mathrm{~nm}$ ) at $250 \mathrm{MHz}$ repetition rate. Figure $1 \mathrm{~b}$ shows the analytical and numerical results of the OPO behavior around the spectral phase transition point, and Fig. 1c,d show the experimental and numerical results of the spectral behaviors of the OPO.

We have shown that OPOs around degeneracy can undergo a spectral phase transition, which we have demonstrated experimentally and verified numerically. Our results shed light on spectral behaviors of a new operation regime of nonlinear photonic resonators which can potentially enable new opportunities for sensing and information processing. The square-root behavior can be a resource for enhanced sensing, similar to exceptional points [5], and the dynamics around the critical point may be utilized for information processing similar to optical Ising machines [7]. Extension of this study to below the oscillation threshold can lead to opportunities for quantum information processing. Such a spectral phase transition can also happen in Kerr OPOs. Recent progress in lithium niobate based integrated devices will enable development of sensors based on phase transition on chip [8].

This work is supported by ARO Grant No. W911NF-18-1-0285 and NSF Grant No. 1846273.

\section{References}

1. M.C. Cross, and P.C. Hohenberg, Reviews of modern physics 65 (3) , (1993).

2. Ş.K. Özdemir, S. Rotter, F. Nori, and L. Yang, Nature materials, 18 (8),(2019).

3. M. Vaupel, A. Maitre, and C. Fabre, Physical Review Letters 83 (25) , (1999).

4. R. Hamerly, A. Marandi, M. Jankowski, M. Fejer, Y. Yamamoto and H. Mabuchi, Physical Review A 94(6) , (2016).

5. H. Hodaei, A. U. Hassan, S. Wittek, H. Garcia-Gracia, R. El-Ganainy, D. N. Christodoulides, and M. Khajavikhan, Nature 548 (7666), (2017).

6. C. Langrock, and M.M. Fejer, Optics letters 32(15) , (2007).

7. A. Marandi, Z. Wang, K. Takata, R.L. Byer, and Y. Yamamoto, 8(12), (2014).

8. C. Wang, M. Zhang, B. Stern, M. Lipson, and M. Loncar, Optics express 26 (1547) , (2018). 